\title{
DEFINICIÓN DE DOS NUEVOS TIPOS DE ÁNFORAS GADITANAS: LAS PUERTO REAL 1 Y 2
}

\section{DEFINITION OF TWO NEW AMPHORAE TYPES FROM CADIZ: PUERTO REAL 1 AND 2}

\author{
por \\ ENRIQUE GARCÍA VARGAS y M. ${ }^{a}$ LUISA LAVADO FLORIDO
}

\begin{abstract}
RESUMEN Se definen en este artículo dos tipos anfóricos romano-gaditanos cuyos ejemplares se incluyen a menudo en otros grupos formales y que se han denominado provisionalmente Puerto Real 1 y 2 , ya que su producción ha sido constatada por ahora tan sólo en el alfar de Puente Melchor, en Puerto Real. Se trata de contenedores dedicados al transporte de salsas de pescado que fueron producidos a caballo entre los siglos II y III d. C. y que se llegaron a exportar vacíos para el abastecimiento de factorías de salazón del otro lado del Estrecho.
\end{abstract}

ABSTRACT Two new types of amphoras from the Roman Gades, provisionally named Puerto Real 1 and Puerto Real 2, are defined in this paper. Included till now in other morfological groups, the PR 1 and PR 2 carried out between the late IInd. and the early IIIrd. centuries some kind of fish sauce, and were sent out emptie ones to fish sauce factories in the oposite coast of the Gibraltar Strait.

\section{1.- INTRODUCCIÓN}

Las páginas que siguen se dedican al estudio y la definición formal de dos tipos anfóricos gaditanos muy próximos entre sí que hemos denominado provisionalmente Puerto Real 1 y Puerto Real 2.

Se trata de dos series anfóricas contemporáneas. De la Puerto Real 2 se han llegado a publicar ejemplares completos (Jiménez Cisneros 1971: Lám LX; Beltrán 1970: fig. 172. 2; Idem 1977: fig. 24.), mientras que las Puerto Real 1 han sido asignadas a menudo a la forma IIb de M. Beltrán. A pesar de que tanto unas como otras se apartan tipológica y cronológicamente de las Beltrán IIb su filiación formal con respecto a éstas es evidente, lo que explica la confusión.

Sólo recientemente se han considerado las Puerto Real 1 como un tipo aparte de las Beltrán IIb (MartinKilcher 1994: 401), a propósito del material anfórico hallado en la antigua Colonia Pia Apollinaris Augusta 
Emerita Raurica (Augst y Kaiseraugst), en Suiza, donde se le ha denominado Augst 30 en lo que es más una nomenclatura interna referida al material anfórico hallado en el lugar que una denominación para el tipo.

Al mismo tiempo que se publicaba el material de Augst, las excavaciones de urgencia dirigidas por $\mathrm{M}^{\mathrm{a}}$ L. Lavado en el alfar de Puente Melchor (Puerto Real, Cádiz) permitían conocer con algún detalle un centro de manufactura (¿el único?) de estos contenedores (García Vargas y Lavado 1995). Ello ha hecho posible no sólo la identificación, sino también la definición de ambos tipos (García Vargas e.p.).

De momento, hemos de contentarnos con una definición preliminar de éstos, más sobre la base de variables formales que cuantitativas, en tanto se aborde el estudio en profundidad de los materiales de Puente Melchor. Con ello no se pretende sino aclarar en lo posible el panorama con respecto a unas producciones poco conocidas y que comienzan a identificarse cada vez con más claridad en los centros de consumo (Bernal y Pérez Rivera, e.p.)

\section{2.- DESCRIPCIÓN}

\subsection{Puerto Real 1 (figs. 1.1 y 2.1-4)}

Se trata de un ánfora con cuerpo de perfil piriforme cuyo diámetro máximo se alcanza en el tercio inferior y cuello bitroncocónico o de tendencia cilíndrica. Las dimensiones de los ejemplares más completos permiten suponer una altura total en torno al metro, mientras que el diámetro máximo de la panza se sitúa entre los 30 y los $40 \mathrm{~cm}$ El grosor máximo de las paredes del cuerpo es de 1.6-1.8 cm. Los diámetros de boca están entre los 18 y los $19.5 \mathrm{~cm}$, con alturas de borde de entre 2 y $3 \mathrm{~cm}$. y grosores máximos en torno a 2.5-3.5 $\mathrm{cm}$. Las asas, con sección de tendencia oval se insertan en el labio y sobre los hombros, suelen medir entre 19.5 y $23 \mathrm{~cm}$., aunque en algunos casos alcanzan los $26 \mathrm{~cm}$. Ningún ejemplar ha conservado la base, sin embargo, son muy frecuentes en los estratos donde aparece el tipo los pivotes cilíndricos con anillo terminal, dándose el caso que uno de ellos se conserva junto al fondo de un ánfora que, por sus dimensiones, puede ser una Puerto Real 1.

Cabe la posibilidad de que algunos de estos pivotes correspondan en realidad a Keay XVI. Sin embargo, los pocos ejemplares completos de Keay XVI gaditanas carecen de anillo terminal (García Vargas, e. p.). Además, los índices de frecuencia de estos pivotes en Puente Melchor se acercan más a los que presentan los bordes de Puerto Real 1 que a los de Keay XVI. Por otra parte, las ánforas el tipo Augst 30 de Augst y Kaiseraugst llevan pivote con anillo terminal (Martin-Kilcher 1994: 401) lo que nos hace albergar pocas dudas acerca de la morfología de éste elemento en las Puerto Real 1.

En Puente Melchor, pueden distinguirse con bastante claridad dos subtipos que difieren en la forma y la longitud del cuello.

El subtipo A (fig. 1.1) presenta cuello bitroncocónico corto $(6-8 \mathrm{~cm}$.) y ancho $(13-15 \mathrm{~cm}$. en su tramo central) que en la transición hacia la panza enlaza con unos hombros relativamente marcados.

El subtipo B (figs. 2. 1-4) se caracteriza por el desarrollo del cuello, esta vez de tendencia cilíndrica, cuya longitud se sitúa entre los 10 y los $14 \mathrm{~cm}$., con una anchura media de $11 \mathrm{~cm}$. En las Puerto Real $1 \mathrm{~B}$ la transición entre cuello y cuerpo es imperceptible, por lo que, a diferencia del subtipo anterior, carecen de hombros. 
Es posible, sin embargo, que bajo la denominación Puerto Real 1B estemos englobando en realidad dos variantes cuya característica común sea la longitud del cuello y la ausencia de hombros, pero que a su vez se diferencien en detalles de la morfología del borde y las asas.

Dado lo fragmentario de los ejemplares hoy por hoy conocidos, no nos aventuramos a disociar de un modo definitivo ambas variantes, por lo que apuntamos tan sólo la existencia de un tercer grupo de Puerto Real 1 (Puerto Real 1B2), en el que habría que incluir gran parte del material de Augst y cuya presencia es, sin embargo, minoritaria en Puente Melchor (fig. 2.4)

\subsection{Puerto Real 2 (figs 1.2 y 3.1-2)}

Un ánfora completa del tipo con la marca SOC se expone en el Museo Arqueológico de Jerez. El origen de este ejemplar en Puente Melchor está garantizado por la marca impresa bajo el asa y por la publicación del mismo como procedente del alfar (Jiménez Cisneros 1971: 150 y lám LX; Beltrán 1977: 111).

La Puerto Real 2 es un ánfora robusta de $85 \mathrm{~cm}$. de altura y 40 de anchura máxima, boca amplia, cuerpo piriforme más ancho en su tercio inferior y base plana rehundida (figs. 1.2 y 3.2). El grosor de las paredes del cuerpo oscila entre 1.5 y $1.7 \mathrm{~cm}$., las asas, con sección de tendencia circular y una longitud media de 20-23 cm., arrancan desde el mismo borde y caen sobre los hombros del recipiente. Los bordes presentan sección rectangular o subrectangular y alcanzan medidas que oscilan entre $20-22 \mathrm{~cm}$. de diámetro, 2.5-3.5 $\mathrm{cm}$. de altura y $3-3.5 \mathrm{~cm}$. de grosor máximo. El cuello es muy ancho, corto y cilíndrico, en torno a los 16-17.5 $\mathrm{cm}$. de longitud y los $7-8 \mathrm{~cm}$. de altura total.

\section{3.- DENOMINACIÓN}

Conviene individualizar ambas formas no sólo desde el punto de vista formal, sino también en su denominación, pues tanto las Puerto Real 1 como las Puerto Real 2 difieren notablemente de las Beltrán IIb, entre las que a menudo se incluyen (últimamente Lagóstena 1996; Pérez López et alii e. p.). El mismo M. Beltrán (1970: 436; 1977: 112) consideró quelas Puerto Real 2, dada la peculiar morfología de su base no eran sino una variante de su forma IIb con el "fondo umbilicado."

El carácter provisional de la definición de ambos tipos y el hecho de que los hallazgos fuera de su lugar de producción sean aún escasos nos han inclinado por una denominación de tipo "geográfico", si bien somos conscientes de que la necesaria reordenación tipológica de las ánforas béticas, que ha de traer aparejada una nomenclatura más homogénea para el material provincial, propiciará la asignación de un nombre más apropiado. Proponemos en tanto Puerto Real 1 y Puerto Real 2, nombres que al menos tienen la ventaja de que remiten inmediatamente a su lugar de producción.

\section{4.- ORIGEN MORFOLÓGICO}

La descripción de un tipo anfórico por extensión, esto es mediante la descripción de los rasgos que comparten todos los ejemplares del mismo, no parece suficiente para ofrecer una definición correcta del mismo. Sin embargo, la imposibilidad de ofrecer una definición por intensidad queda en parte compensada por las "garantías de cientificidad" que presenta la definición genética, esto es, la que considera el origen o causa eficiente del objeto definido (Spinoza, Epist. LX). 
Desde esta perspectiva, las Puerto Real 1 y 2 aparecen como "herederas" de las Beltrán IIb cuyo perfil piriforme continúa siendo uno de los rasgos visualmente predominante en las nuevas formas. Sin embargo, otras características como la menor longitud del cuello, incluso en el subtipo B de Puerto Real 1, y la posición y forma de las asas parecen preludiar a las Keay XVI (Almagro 50 similis), con las que también se han confundido (Lagóstena 1996: 74) y con las que aparecen asociadas en los mismos vertederos de Puente Melchor (fig. 3.3)

Por otra parte, es posible que las ánforas denominadas Lusitana 9 (Dias Diogo 1987: fig. 6, nº9). producidas en el valle del Sado, en Portugal, y cuyo aspecto general recuerda, no sólo por la base plana, a nuestras Puerto Real 2, no sean sino un trasunto o imitación formal de éstas últimas, dado que la Lusitana 9 fue producida en la "fase B" (mitad del s. II d. C. en adelante) de las dos en que A. M. Dias Diogo (1992: 95) ha dividido la vida productiva de los alfares del Sado.

\section{5.- CRONOLOGÍA}

Los ejemplares de Puente Melchor se localizan en los vertidos fechados entre la segunda mitad del s. II d.C. y los primeros años del s. III d.C., de modo que ésta debe ser la fecha de máxima producción de ambos tipos. Son pues, formas características de época severiana, momento en el que parecen haber desplazado a la Beltrán IIb, al menos en Puente Melchor.

Fuera del alfar, tan sólo conocíamos un par de Puerto Real 1 descontextualizadas: una de ellas (subtipo A) hallada en aguas del Estrecho, cerca de Tarifa ${ }^{1}$ y la otra, también de procedencia submarina, procedente de Rigisvilla (Toscana) (M. Martelli et alii (1982), 26 fig. 39), clasificada como Dressel 38-9, es decir, Beltrán IIb. Esta última muestra una morfología muy próxima a la de nuestro subtipo B. A ellas habría que unir un cuello, también sin contexto arqueológico, depositado en el Museo de Ceuta y que corresponde al subtipo 1A (Martínez Maganto et alii 1997: fig. 13, no 530).

Los últimos hallazgos de Augst (Martin-Kilcher 1994: 401-2) y Ceuta (Bernal y Pérez Rivera e. p.) remiten a las mismas fechas de Puente Melchor: fines del s. IÍ, principios del III d. C., para las Puerto Real 1, lo que parece ser el momento de máxima producción de unos tipos cuyo origen puede ser algo anterior y su producción mantenerse algunas décadas durante el s. III d. C., toda vez que la coetanidad de las dos formas tratadas aquí viene sugerida por su hallazgo en los mismos niveles de vertido del alfar.

\section{6.- EPIGRAFÍA}

Las Puerto Real 1 suele ir marcada con un sello bajo el asas y, a veces, un segundo, idéntico, sobre el cuerpo. El repertorio epigráfico del tipo está integrado por las siguientes marcas:

- SOC, en cartela rectangular.

- SOCI, en cartela rectangular.

- CL.SOC, en cartela rectangular.

- BV[], en cartela circular sobre un pivote ¿de PR1?

- L.M.[], en cartela rectangular. ${ }^{2}$

1. Agradecemos la información al Prof. M. Martín Bueno.

2. (M. Beltrán Lloris (1977), fig. 23. Éstas iniciales coinciden con las de L. Mevius Rufus del alfar de Villanueva que se fecha al menos cien años antes (E. García Vargas, e. p.). 
En cuanto a las Puerto Real 2, el ejemplar de Jerez tiene un sello en cartela rectangular bajo el asa en el que se lee SOC. Otro cuello de Puente Melchor lleva una marca sobre el cuello, a la altura del arranque superior del asa con la inscripción FEX en cartela rectangular (fig. 3.1).

\section{7.- CONTENIDO}

La forma general del envase remite a las producciones salsarias altoimperiales (Beltrán IIb, aunque hoy sabemos que las Beltrán IIb también llevaron vino y defrutum: García Vargas e. p.) y bajoimperiales (Keay XVI).

La dotación epigráfica de ambos tipos no es, sin embargo, concluyente: los sellos SOC y SOCI no tienen que hacer referencia necesariamente a una societas conservera, mientras que el único titulus conocido sobre Puerto Real 1b, hallado en Augst, señala Lacca(tum) (Martin-Kilcher 1994: 406, no P30).

La naturaleza del laccatum es contradictoria: S. Isidoro (Orig. 20.3.10) afirma que se trata de una bebida hecha con leche, y sin embargo los comentaristas modernos han sugerido otros productos.

H. Dressel lo considera un tipo de vino sazonado con lacca, una hierba medicinal (Dressel, 1879:67-9), aunque posteriormente se inclina por identificarlo como una salsa, también sazonada con lacca (CIL XV 4735); para Zevi (1966: 243), la forma del ánfora indica un contenido salsario, mientras que Callender (1965: 18) cree que se trata de vino. En Roma, las inscripciones de $C I L X V$ que señalan laccatum aparecen sobre Dressel 7 y 8 , lo que parece indicar que se trata de una salsa, puesto que hasta hoy, no se conoce inscripción vinaria alguna sobre estos tipos.

Tal vez el indicio más claro de que las Puerto Real 1 y 2 son ánforas salsarias es su hallazgo de Puerto Real 1 con sello SOC en el contexto de una factoría de salazones de Ceuta, hacia donde parecen ser enviadas vacías (Bernal y Pérez Rivera e. p.). Esto, si no es que estamos ante un nuevo caso de contenedor bivalente, situaría sin muchas dudas al laccatum entre las salsas de pescado.

\section{8.- CENTROS DE PRODUCCIÓN}

Puente Melchor, en la costa de Puerto Real, concretamente a ambos lados del PK. 667 de la N. IV, es hoy por hoy el único centro conocido que produjo la forma. El topónimo Puente Melchor es reciente en la bibliografía sobre hornos anfóricos (Lazarich et alii 1991: 98, nํ11; Pérez López et alii, e. p.; García Vargas, e. p.; Idem y Lavado 1995; Lagóstena 1996). El alfar, es sin embargo conocido desde 1946 con el nombre de Paso a nivel (Pemán 1959: 169 y fig. 1) por haberse hallado restos anfóricos en la żona con motivo de la construcción del puente que sustituyera al antiguo paso a nivel de la vía del ferrocarril Madrid-Cádiz. $\mathrm{M}^{\mathrm{a}}$ J. Jiménez Cisneros designó este alfar de manera diferente en cada ocasión: “....antiguo paso a nivel del ferrocarril” (1971: 149), “...puente... en la carretera, antes de llegar a Puerto Real (1971: 150), Paso a nivel (1971: lám. LXIII), Huerta paso a nivel (Lám. LXIV), mientras que M. Beltrán lo llamó Villanueva paso a nivel del ferrocarril (1977: 111), y D.P.S. Peacock lo reseñó con su nombre original de Pasó a nivel (1974: 329).

\section{9.- TECNOLOGÍA}

Las ánforas Puerto Real 1 y 2 presentan una aspecto tosco y poco cuidado. Son ánforas muy robustas cuyas líneas apenas se tratan de suavizar, por lo que las marcas de torno y las líneas de unión suelen.ser evidentes. Aún así, no son raros los alisados en la intersección de piezas. Los ejemplares de ambos tipos 
están confeccionados con pastas de cocción oxidada continua, duras y no muy porosas, de fractura irregular y colores rojo claro (M 37), rosa (M 69, M 70) amarillo (L 90) y verde en fallos de cocción (M 89) ${ }^{3}$, arenosas, con partículas dispersas de mineral de hierro de más de $5 \mathrm{~mm}$ y a veces ligeros engobes blanquecinos en la superficie. Al microscopio, se distingue una matriz de arcilla que incluye hasta un $20 \%$ de arena y en cuyo seno se aprecian inclusiones no muy abundantes de cuarzo subredondeado o redondeado y, en menor cantidad, subangulares de feldespato, de entre 0.03 y $0.05 \mathrm{~mm}$. También se encuentran presentes fragmentos de calcita, mica, plagioclasasas y foraminifera, así como arenisca.

\section{0.- CONCLUSIONES}

La definición, aún a titulo provisional, de las Puerto Real 1 y 2 como tipos independientes rescata del anonimato dos series anfóricas de las que se conocían ejemplares aislados casi siempre mal clasificados.

La asignación de una cronología ajustada y la localización en la bahía de Cádiz del hasta hoy único centro productor de ambos tipos son hechos del mayor interés, no sólo desde una perspectiva tipológica, sino también a efectos del análisis histórico.

En efecto, los contextos tipológico y epigráfico en que se incluyen las Puerto Real 1 y 2 en los vertederos del alfar de Puente Melchor (García Vargas y Lavado 1995) apuntan a una producción en masa como punto de llegada de un proceso de "industrialización" de los alfares de la bahía gaditana, proceso que no podemos detenernos a analizar ahora, pero que ha sido estudiado por nosotros con anterioridad (García Vargas 1996; Idem e. p.).

La culminación del proceso, unida a la relativa variedad de tipos producidos en Puente Melchor que incluyen ánforas vinarias y olearias (García Vargas y Lavado 1995), son el mejor testimonio de una cierta reactivación económica en la Bahía para unas fechas que oscilan entre los últimos años del siglo II y los primeros del III d. C. y que caen de lleno en los momentos iniciales de la dinastía severiana.

No parece, pues, que sea ajena a dicha reactivación, cuyo reflejo en otros puntos de la costa Bética es evidente (Bernal 1997; Gener et alii 1993), la "política económica" de Septimio Severo, una política que pivotó sobre la reforma del sistema monetal y entre cuyos resultados destaca el aumento global de los bienes en circulación (Mazza 1973), muchos de los cuales se envasaron en ánforas. Ello consiguió contener durante algún tiempo las tendencias inflacionistas que comenzaban a marcarse en la economía imperial.

La ánforas de Puente Melchor ilustran bien este aumento de los bienes circulantes con respecto al periodo inmediatamente anterior, un incremento que no se refleja en los gráficos realizados sobre barcos sumergidos porque las ánforas béticas de época severiana sólo comienzan a ser conocidas ahora (García Vargas e. p.). De entre ellas, las Puerto Real 1 y tal vez las Puerto Real 2, cuya producción es más corta, reflejan especialmente dicha situación y, en concreto, un aspecto aún mal estudiado: el comercio de envases vacíos con destino a las fábricas de salazón (Bernal, e. p.), no solo de la costa cercana.

Efectivamente, las últimas investigaciones en Ceuta (Bernal y Pérez Rivera, e. p.) documentan por primera vez el empleo de ánforas Puerto Real 1 con marca SOC procedentes de Puente Melchor para el envasado de las conservas marinas locales entre finales del s. II y principios del III d. C., fechas que coinciden con las que ofrece el alfar. Las Puerto Real 1 son las únicas empleadas con este cometido en Ceuta lo que abre interesantes perspectivas al estudio de las formas de producción de salsamenta y salsas de pescado en época medioimperial, un tema fuertemente ligado al que nos ocupa, pero que debe ser tratado en otro lugar.

3. Todas las referencias remiten al Code des colours des sols de E. Cailleaux (1976). 


\section{BIBLIOGRAFÍA}

BELTRÁN LLORIS, M. (1970): Las ánforas romanas en España, Zaragoza.

(1977): "Problemas de la morfología y del concepto histórico-geográfico que recubre la noción tipo. Aportaciones a la tipología de las ánforas béticas", Méthodes classiques et méthodes formelles dans l'étude des amphores. Actes du Colloque de Rome, 27-29 Mai 1974. Coll. del I'́cole Française de Rome 32 Roma: 97-31.

BERNAL CASASOLA, D; (1997): "Novedades de epigrafía anfórica en la Bética. Talleres costeros granadinos del Bajo Imperio" Boletín de la Asociación Española de Amigos de la Arqueología 37: 99-110.

- (en prensa): "Transporte de envases vacíos en época romana: a propósito de dos talleres anfóricos béticos de época alto (El Rinconcillo, Algeciras, Cádiz) y bajoimperial (Los Matagallares, Salobreña, Granada)" II Congreso de Arqueología Peninsular, Zamora, 1996.

- y PÉREZ RIVERA, J. (en prensa): La factoría de salazones de Septemfratres" Homenaje a Carlos Posac. Instituto de Estudios Ceutíes. Ceuta.

CALLENDER, M. H. (1965): Roman Amphorae with an index of stamps. Londres.

DIAS DIOGO, A. M. (1987): "Quadro Tipologico das ânforas de fabrico lusitano", AP 4: 179-91.

_ (1992): "Elementos para a caracterização e periodização da economia do Baixo Sado, durante a epoca romana", Arqueología hoje I: 92-100.

DRESSEL, H. (1879): "Di un grande deposito di anfore rinvenuto nel nuovo quartiere del Castro Pretorio" Bullettino della Commissione Archeologica Comunale di Roma 36-112.

- (1899): Corpus Inscriptionum Latinarum, XV, 2: Inscriptiones Urbis Romae Latinae. Instrumentum domesticum. Partis posterioris fasciculus I. Berlin.

GARCÍA VARGAS, E. (1996): "Las ánforas del alfar de "El Gallinero" (Puerto Real, Cádiz) en el contexto de las producciones anfóricas gaditanas" Terceras Jornadas de Historia de Puerto Real (Puerto Real 1995, Puerto Real 1996) 33-81.

- (en prensa): La producción de ánforas en la bahía de Cádiz en época romana (siglos II a.C.-IV d.C). - y LAVADO FLORIDO, Ma. L. (1995): “Ánforas alto, medio y bajoimperiales producidas en el alfar de Puente Melchor (= Villanueva, paso a nivel: Puerto Real, Cádiz)”, Spal 4: 215-228.

GENER VASALLOTE, J. Ma., MARFIL RUIZ, P. F., PUENTEDURA BÉJAR, M., (1993): "Loma de Ceres, un centro de producción anfórico" Actas del II Congreso Peninsular de Historia Antigua (Coimbra 1990) 973-993.

JIMÉNEZ CISNEROS, M. Ja . (1971): Historia de Cádiz en la Antigüedad. Cádiz.

LAGÓSTENA BARRIOS, L. (1996): Alfarería romana en la bahía de Cádiz. Cádiz.

LAZARICH, M., ALONSO, M‥C., LADRÓN DE GUEVARA, I., SÁNCHEZ ANDREU, M., RODRÍGUEZ DE ZULOAGA, M. (1991): "Informe preliminar de la primera campaña del proyecto de prospección arqueológica sistemática de la campiña sur gaditana: Término de Puerto Real”, Anuario Arqueológico de Andalucía'89 II, Sevilla: 98-100.

MARTELLI, A. et alii (1982): "Archeologia subacquea in Toscana”, Archeologia Subacquea, Bolletino d'Arte, supp. 4 (1982).

MARTÍNEZ MAGANTO, J., GARCÍA JIMÉNEZ, R., BERNAL CASASOLA, D. (1997): Ánforas del Museo de Ceuta, Ceuta.

MARTIN-KILCHER, St. (1994): Die Römischen Amphoren aus Augst und Kaiseaugst. Forschungen in Augst 7/2. Augst.

MAZZA, M (1973): Lotte sociale e restaurazione autoritaria nel III secolo d. Chr, Roma-Bari. 
PEACOCK, D. P. S. (1974): “Amphorae and the Baetican fish industries”, Antiquaries Journal 54: 232-43. PEMÁN, C. (1959): "Alfares y embarcaderos romanos en la provincia de Cádiz”, AEspA XXXII: 169-73. PÉREZ LÓPEZ, I., LAZARICH, Ma., ALONSO, Ma . C., LADRÓN DE GUEVARA, I., RODRÍGUEZ DEZULOAGA, M., SÁNCHEZ ANDREU, M. (en prensa) "Yacimientos litorales del Término Municipal de Puerto Real (II). Puente Melchor" II Encuentro Internacional de Arqueología del Suroeste de la Península Ibérica. Faro, 7-9 de noviembre de 1996.

ZEVI, F. (1966) "Appunti sulle anfore romane. La tavola tipologica del Dressel” Archeologia Classica XVIII. 

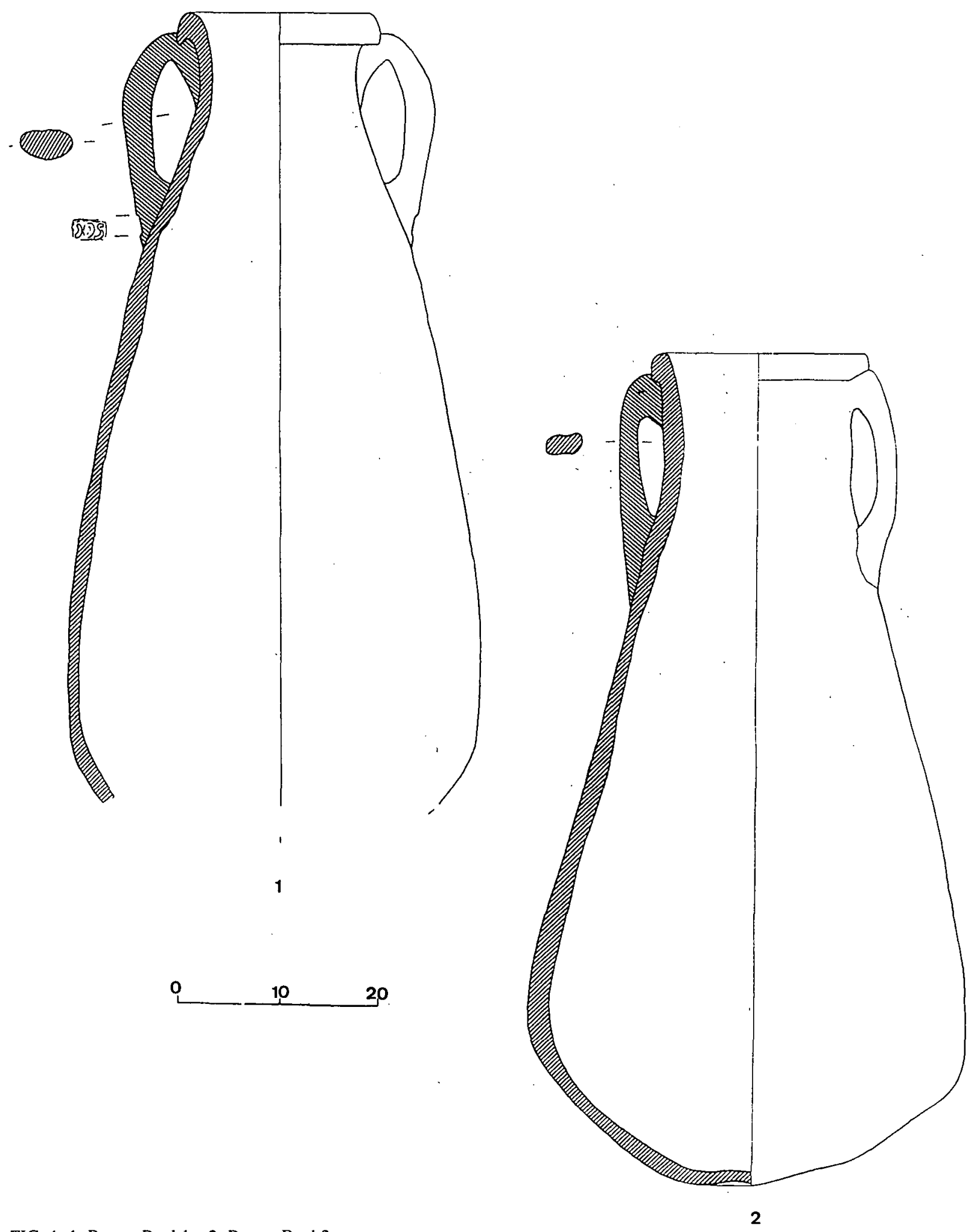

FIG. 1. 1: Puerto Real 1a. 2: Puerto Real 2. 


$$
\begin{aligned}
& 94 \\
& 89
\end{aligned}
$$




$$
\begin{aligned}
& d \\
& p
\end{aligned}
$$

